Trauma Berufskrankh 2007 · 9[Suppl 3]:

S336-S338

DOI 10.1007/s10039-007-1233-4

Online publiziert: 6. Juni 2007

(c) Springer Medizin Verlag 2007
F. Ritter

Landesverband Südwestdeutschland

der gewerblichen Berufsgenossenschaften, Heidelberg

\title{
Organisationsformen im Heilverfahren aus Sicht der BG-Administration
}

\section{Heilbehandlung „mit allen geeigneten Mitteln"}

Die allgemein bekannte Tatsache, dass in der gesetzlichen Unfallversicherung Heilbehandlung mit allen geeigneten Mitteln durchzuführen ist, ist dem Umstand zu verdanken, dass - historisch gewachsen - die gesetzliche Unfallversicherung die Ablösung der Unternehmerhaftpflicht darstellt. Deswegen ist es Pflicht des Unfallversicherungsträgers, nach Eintritt von Arbeitsunfällen oder Berufserkrankungen die Gesundheit und die Leistungsfähigkeit der Versicherten mit allen geeigneten Mitteln wiederherzustellen und sie oder ihre Hinterbliebenen durch Geldleistungen zu entschädigen ( $\$ 1$ Satz 1 Nr. 2 SGB VII [2]). Dieser Qualitätsmaßstab ist höher, als beispielsweise im Bereich der gesetzlichen Krankenversicherung. Das Sozialgesetzbuch V (SGB V) beschreibt hier die Leistungen der Krankenversicherung als Mittel, die den Versicherten unter Beachtung des Wirtschaftlichkeitsgebots zur Verfügung gestellt werden, soweit diese Leistungen nicht der Eigenverantwortung der Versicherten zugerechnet werden. (...) Krankenkassen, Leistungserbringer und Versicherte haben darauf zu achten, dass die Leistungen wirksam und wirtschaftlich erbracht und nur im notwendigen Umfang in Anspruch genommen werden ( $\$ 2$ Abs. 1 und 4 sowie $\$ 12$ Abs. 1 SGB V [1]).

Gerade in dem jetzt vorherrschenden Reformprozess der gesetzlichen Unfallversicherung ist immer wieder verdeutlicht worden, dass die Bundesrepublik
Deutschland durch diese Ablösung der Unternehmerhaftpflicht, die untrennbar mit einem höheren Maßstab einer „Schadensregulierung " als in den anderen Sozialversicherungsbereichen verbunden ist, mit den gesetzlichen Unfallversicherungsträgern über eine gut funktionierende Institution verfügt, die besonders den sozialen Frieden zwischen Arbeitgebern und Arbeitnehmern sichert [3]. In dem aktuellen Entwurf des 2. Teils des UVRG zur Reform des Leistungsrechts der gesetzlichen Unfallversicherung hält das Bundesministerium für Arbeit und Soziales an der Rehabilitation mit allen geeigneten Mitteln fest, ja betont sogar besonders die Nachrangigkeit der Rentenzahlung gegenüber einer entsprechenden intensiven Rehabilitation. Die Anreize für den Unfallversicherungsträger, Rehabilitation mit allen geeigneten Mitteln durchzuführen, sollen erhöht werden [4].

\section{Sicherstellung der Versorgungsqualität durch die GUV}

- Durch welche Anforderungen stellt der Unfallversicherungsträger sicher, dass er dem oben angeführten hohen Qualitätsmaßstab im Rehabilitationsprozess gerecht wird?

- Worin besteht der Unterschied zur Heilbehandlung nach dem Maßstab des „medizinisch Notwendigen“?

Die gesetzliche Unfallversicherung braucht den besonderen Status der am berufsgenossenschaftlichen Verfahren be- teiligten Ärzte. Der in den jeweiligen Anforderungen der gesetzlichen Unfallversicherungsträger nach $\$_{34}$ SGB VII festgeschriebene Maßstab an die fachliche Befähigung, die personelle und die sächliche Ausstattung sowie die Pflichten dient nicht dem Selbstzweck. Er stellt sicher, dass eine hochwertige Heilbehandlung erfolgt und der gesetzliche Auftrag erfüllt wird.

Dieser Qualitätsmaßstab rechtfertigt auch die Verknüpfung des Facharztstatus des „Chirurgen“ oder des „Orthopäden und Unfallchirurgen“ mit der Schwerpunktbezeichnung „Unfallchirurgie“ oder die Zusatzbezeichnung "spezielle Unfallchirurgie“, denn erst hierdurch wird die besondere unfallchirurgische Behandlung gewährleistet und darum geht es beim Arbeitsunfall! Der immer wieder aufkommende Vorwurf, die Unfallversicherungsträger würden durch ihre hohen Anforderungen ihr Versorgungsnetz ausdünnen und damit gefährden, ist durch nichts belegt. Im Gegenteil, die Zusammenarbeit mit der gesetzlichen Unfallversicherung ist nach wie vor für einen Krankenhausträger und für einen niedergelassenen Arzt ein begehrtes und lukratives Geschäft. Das beweisen die weiterhin hohen Zulassungszahlen der Landesverbände der gewerblichen Berufsgenossenschaften beim D-Arzt-, H-Arzt- und VAV-Verfahren (s. z. B. [5]).

Die Qualitätsmaßstäbe werden in einem dauerhaften Dialog mit den Medizinern immer wieder kontrolliert. Eine interdisziplinäre Arbeitsgruppe, bestehend aus Mitarbeitern der Unfallversicherungsträger und Ärztlichen Direktoren der BG- 
Unfallkliniken, prüft dauerhaft die Anforderungen und festgeschriebenen Zuweisungspflichten auf ihre Aktualität und Effektivität.

Heilbehandlung mit allen geeigneten Mitteln fängt nach dem Verständnis der gesetzlichen Unfallversicherung am Unfallort an. Deswegen beginnt dort mit dem Notarzt der hohe Qualitätsmaßstab. Der dann erstbehandelnde Arzt im Krankenhaus oder in der Praxis muss besonders qualifiziert sein. Bei schwereren Verletzungen muss Facharztbehandlung entsprechend den Anforderungen der gesetzlichen Unfallversicherung erfolgen. Eine Delegation von Verantwortung an einen Mitarbeiter oder Kollegen eines D- oder $\mathrm{H}$-Arztes gestattet die gesetzliche Unfallversicherung nicht. Verantwortung bedeutet dabei, die Qualität bei sich selbst, dem Personal, der apparativen und der sächlichen Ausstattung zu sichern. Wie soll ein Arzt unmittelbar einen Arbeitsunfallpatienten behandeln, wenn er sich erst zu seinem angemieteten Operationszentrum begeben muss? Outsourcing gefährdet die unmittelbare ganzheitliche Diagnostik und Behandlung des Unfallverletzten in der Verantwortung des DArztes. Das gilt beim niedergelassenen Arzt ebenso wie im Krankenhaus.

Die Landesverbände der gewerblichen Berufsgenossenschaften kennen die Schwierigkeiten im Krankenhaus bei der persönlichen Leistungserbringung des Durchgangsarztes. Deswegen ist es möglich, Ständige Vertreter bei Krankenhäusern, die zum Verletzungsartenverfahren zugelassen sind, über die Landesverbände zu benennen. Hier gibt es übrigens keine zahlenmäßige Begrenzung, sondern eine Orientierung an einer vernünftigen Organisationsstruktur in der jeweiligen Klinik. Aber so ist Qualität sichergestellt, denn jemand, der die erforderliche Fachkunde nicht hat, darf auch nicht den durch einen Arbeitsunfall verletzten Patienten behandeln.

\section{Resümee}

Gerade diejenigen Ärztinnen und Ärzte, die an dem besonderen berufsgenossenschaftlichen Heilverfahren mit allen geeigneten Mitteln beteiligt sind, sollten nicht Gründe suchen, dieses System an

Trauma Berufskrankh 2007 · 9[Suppl 3]: S336-S338 DOI 10.1007/s10039-007-1233-4

(c) Springer Medizin Verlag 2007

\section{F. Ritter \\ Organisationsformen im Heilverfahren aus Sicht der BG-Administration}

\section{Zusammenfassung}

Die gesetzlichen Unfallversicherungsträger haben nach dem Sozialgesetzbuch VII alle Maßnahmen zu treffen, durch die eine (...) sachgemäße Heilbehandlung und, so weit erforderlich, die besondere unfallmedizinische Behandlung gewährleistet werden. Dabei ist die Heilbehandlung "mit allen geeigneten Mitteln" Maßstab. Im Einzelfall bestimmen die gesetzlichen Unfallversicherungsträger die Art, den Umfang und die Durchführung der Heilbehandlung sowie die Einrichtungen, die diese Leistungen erbringen, nach pflichtgemäßem Ermessen. Dabei prüfen sie

\section{Organisational forms in treatments from the viewpoint of those in administration at the employers' liability insurance associations}

\section{Abstract}

The legally required accident insurance institutions are required by the Code of Social Law (SGB) VII to take all steps needed to ensure appropriate treatment and, if required, special trauma. The standard to be applied is treatment "by all appropriate means". The insurance institutions determine these in the individual case after due consideration of the circumstances, with reference to the type, extent and implementation of the treatment and to the units that can supply it. They also test what benefits are appropriate and rea- auch, welche Leistungen geeignet und zumutbar sind. Diesem gesetzlich festgelegten Maßstab hat sich alles Handeln des Unfallversicherungsträgers unterzuordnen. Nur solche Organisationsformen, die diesem Maßstab entsprechen, darf der Unfallversicherungsträger zulassen bzw. nutzen.

\section{Schlüsselwörter}

Heilbehandlung · Berufsgenossenschaften . Durchgangsarzt · Verletzungsartenverfahren . Sozialgesetzbuch VII sonable. All they do must be subject to this legally prescribed standard. The accident insurance is now entitled to approve or use any organisational forms except those that comply with this standard.

\section{Keywords}

Treatment · Employers' liability insurance Procedures by type of injury - Ode of Social Law (SGB) VII associations - Emergency doctor on call . 
die Anforderungen anderer Sozialversicherungssysteme anzupassen. Sie sollten bedenken, dass sie wesentlich dazu beitragen, dass ein solches Heilverfahrensniveau in der Bundesrepublik Deutschland angeboten werden kann. Denn die gesetzliche Unfallversicherung ist nur so gut, wie die für sie tätigen medizinischen Leistungserbringer.

Besondere Zuweisungspflichten, budgetunabhängige Behandlungsfreiheit und ein besonderes Liquidationsrecht lassen sich jedoch nur rechtfertigen, wenn dafür auch eine hohe Qualität garantiert wird. Diesen Gestaltungsspielraum, den die Politik aus gutem Grund weiterhin erhalten möchte, sollten alle Beteiligten zum Wohle unserer Versicherten und $\mathrm{Pa}$ tienten nutzen.

\section{Korrespondenzadresse}

\section{F. Ritter}

Landesverband Südwestdeutschland der gewerblichen Berufsgenossenschaften, Postfach 101480,

69004 Heidelberg

fritter@heidelberg.lvbg.de

Interessenkonflikt. Der korrespondierende Autor gibt an, dass kein Interessenkonflikt besteht.

\section{Literatur}

1. Aichberger F (2005) Sozialgesetzbuch, Textsammlung. Beck, München

2. Bereither-Hahn W, Mehrtens G (2006) Gesetzliche Unfallversicherung. Siebtes Buch Sozialgesetzbuch - Handkommentar. Schmidt, Berlin

3. Bund-Länder-Kommission (2006) Eckpunktepapier der Bund-Länder-Kommission vom 11. Juli 2006. BT-Ausschussdrucksache 16(11)340

4. Bundesministerium für Arbeit und Soziales (2007) Arbeitsentwurf eines Gesetzes zur Reform der gesetzlichen Unfallversicherung (UVRG) vom 27.04.2007. Bundesministerium für Arbeit und Soziales, Berlin

5. Landesverband Südwestdeutschland der gewerblichen Berufsgenossenschaften (2006) Jahresbericht 2005. Landesverband Südwestdeutschland der gewerblichen Berufsgenossenschaften, Heidelberg 